\title{
AVALIAÇÃO DA FUNCIONALIDADE E DA FORÇA DINAMOMÉTRICA LOMBAR DE MINEIROS DO CARVÃO
}

\section{Evaluation of Lumbar Dynamometric Strength and functionality of Coal Miners}

Ana Laura Milanez Marcelo ${ }^{1}$, Maicon da Silva Martins ${ }^{2}$, Willians Cassiano Longen ${ }^{3}$

${ }^{1}$ Fisioterapeuta graduada pela Universidade do Extremo Sul Catarinense - UNESC. Voluntária do Núcleo de Estudos e Pesquisas em Saúde do Trabalhador-NEPST.

${ }^{2}$ Estudante do Curso de Fisioterapia da UNESC. Bolsista de Iniciação Científica pelo PIC-170 do Núcleo de Estudos e Pesquisas em Saúde do Trabalhador-NEPST.

3 Fisioterapeuta. Professor e Pesquisador do Programa de Pós-Graduação em Saúde Coletiva-PPGSCol da UNESC. Coordenador do NEPST.

\section{Endereço para correspondência:}

Willians Cassiano Longen

Clínicas Integradas - Laboratório de Biomecânica - LABIOMEC

Av. Unversitária, 1105

Bairro: Pinheirinho, Criciúma-SC

CEP: 88806-000

Email: labiomec@unesc.net 


\title{
Resumo
}

O objetivo do estudo foi avaliar a força dinamométrica lombar de mineiros que atuam no subsolo de mina de carvão, correlacionando com Dor, Força e Funcionalidade. O estudo realizado foi uma pesquisa transversal quantitativa de levantamento de campo em uma mina de carvão. A amostra foi composta por 40 mineiros. Foi realizada uma avaliação física funcional com o questionário Owestry Low Back Pain para avaliar a funcionalidade e um dinamômetro lombar japonês da marca Takei ${ }^{\circledR}$. Os resultados obtidos foram de $77,5 \%$ com fraqueza lombar, pouca alteração na funcionalidade com $97,5 \%$ dos mineiros apresentando grau de incapacidade mínima. Pode-se concluir que os mineiros do subsolo de carvão apresentaram fraqueza lombar, independente da presença ou não de lombalgia mecânica. Esta fraqueza não mostrou correlação estatística com queda da funcionalidade e presença e intensidade de dor. Os achados apontam para a necessidade de acompanhamento destas populações trabalhadoras visando a manutenção plena de suas condições funcionais e livres de afecções musculoesqueléticas da coluna lombar, que podem iniciar-se por sinais como redução da força.

Palavras-chave: Lombalgia mecânica; Dor lombar; Mineiros.

\begin{abstract}
The objective of this study was to evaluate the strength of miners working lumbar dynamometric underground coal mine, correlating with Pain, strength and functionality. The study was a cross-sectional survey of quantitative research field in a coal mine. The sample was composed of 40 miners. Physical functional evaluation was performed with the Oswald questionnaire Low Back Pain to evaluate the functionality and a lumbar dynamometer Japanese branded Take $i^{\circledR}$. The results obtained were of $77.5 \%$ with weakness, low lumbar change in functionality with $97.5 \%$ of miners showing minimal disability grade. It can be concluded that the underground coal miners showed weakness lumbar, independent of the presence or absence of mechanical back pain. This weakness showed no statistical correlation with drop of functionality and the presence and intensity of pain. The findings point to the need for monitoring of these populations to full maintenance workers of their
\end{abstract}


Artigo Original

Saúde Funcional

functional conditions and musculoskeletal disorders-free of the lumbar spine, which can be initiated by signs such as reduced strength.

Keywords: Non-Specific Low Back Pain; Back Pain; Miners.

\section{INTRODUÇÃO}

Pode-se caracterizar a lombalgia como uma dor situada abaixo da margem das últimas costelas e acima das linhas glúteas inferiores. Em relação ao tipo podese classificá-la como mecânica inespecífica ou específica, não mecânica e psicogênica. Em relação ao tempo de duração do sintoma doloroso, pode-se classificá-la como sendo aguda quando conta com o início rápido e duração inferior a quatro semanas, subaguda tendo duração de quatro a doze semanas e crônica quando com duração superior a doze semanas ${ }^{1,2}$.

A lombalgia deve ser encarada como um importante desafio de saúde pública, especialmente por ser considerada uma afecção potencial para de $70 \%$ a $80 \%$ da população em alguma fase da vida. Seu impacto socioeconômico e coletivo torna-se mais expressivo ao envolver populações em idade economicamente ativa, podendo gerar incapacidade e consequentes afastamentos das atividades laborativas ${ }^{2,3}$.

Para diferenciar lombalgia de lombociatalgia é necessário saber se a dor ultrapassa o joelho com indício de envolvimento de uma raiz nervosa, que quase sempre envolve L5 ou S1, denotando irradiação para os membros inferiores, correspondendo assim a um quadro de lombociatalgia 4 .

A lombalgia crônica inespecífica ou lombalgia mecânica comum é caracterizada por não ter um componente estrutural alterado e não possuir uma causa específica. A maioria dos casos de lombalgia tem incidência maior em trabalhadores submetidos a esforços físicos elevados, posturas estáticas frequentes, movimentos repetidos e levantamento de peso ${ }^{1}$. A morbidade mostra-se associada diretamente com causas de aposentadoria por invalidez, sendo responsável por um grande número de auxílio doença via Previdência Social do Brasili ${ }^{5}$.

A lombalgia crônica inespecífica acarreta uma diminuição no controle postural causada diretamente pelas alterações proprioceptivas e alterações de força 


\section{Artigo Original}

\section{Saúde Funcional}

musculares comparada ao indivíduo saudável. Com a diminuição de força ocorre fadiga da musculatura lombar ${ }^{3}$.

A força da musculatura lombar é objetivo da grande maioria dos programas de exercícios para manejo da lombalgia e recuperação funcional. A presença da fraqueza muscular é proveniente de desequilíbrios musculares que influenciam diretamente na qualidade de vida do cidadão. Para a prevenção e reabilitação de desordens musculoesqueléticas é necessário o fortalecimento desses músculos, procurando desenvolver o controle muscular ideal para restaurar e manter a estabilidade funcional do complexo lombo-pélvico ${ }^{6,7}$.

Portanto, a força muscular da coluna lombar é um elemento preditivo de risco, bem como a fraqueza muscular da musculatura lombo pélvica é uma característica por vezes presente no indivíduo lombálgico. O objetivo deste trabalho foi avaliar a força dinamométrica lombar de mineiros de subsolo do carvão correlacionando com dor, força e funcionalidade.

\section{MÉTODOS}

Foi realizado um estudo transversal quantitativo de levantamento de campo em uma mina de carvão.

A amostra aleatória simples foi composta por 40 mineiros do carvão da Mina de Carvão Cruz de Malta, município de Treviso/SC, que apresentavam lombalgia caracterizada como mecânica.

Após a aprovação no Comitê de Ética em Pesquisa (CEP) da UNESC (341.077/2013) foi realizada uma entrevista direta estruturada e avaliações físicofuncionais da coluna lombar, com um instrumento de incapacidade e com a avaliação da força lombar através de um dinamômetro. A entrevista apurou dados de identificação como: nome, idade, sexo, escolaridade, profissão, tempo de serviço na ocupação atual, ocupação anterior e tempo de serviço. Foi aplicado o Owestry Low Back Pain para avaliar o nível de capacidade e incapacidade relacionado à coluna lombar dos mineiros do carvão.

Os procedimentos de coleta de dados foram realizados na Mina Cruz de Malta com participação voluntária dos mineiros após terem sido esclarecidos os objetivos e procedimentos do estudo, mediante a assinatura do Termo de 


\section{Artigo Original \\ Saúde Funcional}

Consentimento Livre e Esclarecido (TCLE). A coleta dos dados ocorreu no mês de março de 2014.

Posteriormente, os dados foram tabulados e analisados no programa Statistical Package for the Social Sciences - SPSS, versão 17.0. O teste de normalidade utilizado foi o Shapiro-Wilk. Para associação entre as variáveis qualitativas Força Muscular, Funcionalidade e Dor foi utilizado o teste estatístico exato de Fisher. Para avaliar a correlação entre a variável tempo de serviço e a força muscular foi utilizado o teste estatístico correlação de Pearson.

\section{RESULTADOS}

Para a realização deste estudo, participaram 40 indivíduos do sexo masculino. Com relação à idade, esta teve uma mediana de 29,5. Já o tempo de serviço em meses apresentou uma mediana de 36,0. A força muscular avaliada na dinamometria mostrou uma mediana de 117,5. A avaliação de funcionalidade mostrou pouca alteração nesta amostra, sendo que houve prevalência de incapacidade mínima. O estudo relacionado com a força dinamometria lombar mostrou que 31 dos participantes apresentaram fraqueza muscular e 9 destes apresentaram uma força muscular dentro da normalidade, baseado nos valores de referência da dinamometria lombar correspondente a idade. Na análise da dor observou-se que 10 mineiros apresentaram dor e 30 não possuíam queixas álgicas (Tabela 1).

Tabela 1. Características Gerais da Amostra.

\begin{tabular}{lc}
\hline Variável & Mediana (amplitude) ou $\mathbf{n}(\%)$ \\
$\mathbf{n}=\mathbf{4 0}$
\end{tabular}


Artigo Original

Saúde Funcional

Variável

Mediana (amplitude) ou $\mathrm{n}(\%)$

$\mathrm{n}=\mathbf{4 0}$

Dor
Ausente
$30(75,0)$

Presente

$10(25,0)$

\section{Fonte: Próprio Autor}

Caracterização do perfil etário e de serviço da amostra. Força dinamométrica lombar em Kgf.

Funcionalidade através do índice de incapacidade - ODQ = Owestry Low Back Pain. Presença de dor.

Em relação à intensidade da dor lombar nos mineiros sintomáticos, houve predomínio de leve e moderada. Foi observado que 5 mineiros apresentaram dor de intensidade leve, 3 de intensidade moderada e 2 com intensidade grave (Tabela 2).

Tabela 2. Intensidade da Dor.

\begin{tabular}{lc}
\hline Variável & $\mathbf{n}(\%)$ \\
$\mathbf{n}=\mathbf{1 0}$ \\
\hline Leve & $5(50,0)$ \\
Moderada & $3(30,0)$ \\
Grave & $2(20,0)$ \\
\hline
\end{tabular}

Fonte: Próprio Autor

Intensidade da dor dos voluntários sintomáticos e faixas de leve, moderada e grave na EVA.

Foi realizada análise de associação entre a força muscular, funcionalidade e dor, sendo que não houve significância estatística $(p>0,66)$ entre estas variáveis analisadas em cruzamento (Tabela 3 ).

Tabela 3. Análise de Associação entre Força Lombar, ODQ e Dor.

\begin{tabular}{llcl}
\hline & Fraqueza & $\begin{array}{c}\text { Dinamometria } \\
\text { Normal }\end{array}$ & Valor-p \\
\hline ODQ & & $9(100,0)$ & 0,999 \\
Mínima & $30(96,8)$ & $0(0,0)$ & \\
Moderada & $1(3,2)$ & & \\
DOR & & $6(66,7)$ & 0,665 \\
Não & $24(77,4)$ & $3(33,3)$ & \\
Sim & $7(22,6)$ & & \\
\hline
\end{tabular}

Fonte: Próprio Autor

Verificação de correlações entre força lombar, funcionalidade e dor.

O resultado referente à correlação entre o tempo de serviço e a força muscular não demonstrou significância estatística $(r=0,031 ; p=0,851)$. 
O estudo revelou que $25 \%$ dos mineiros do carvão que atuam no subsolo, envolvidos neste estudo, apresentaram dor lombar. Conforme achados na literatura, a dor lombar possui um predomínio na população geral em adultos, principalmente em comunidades de trabalhadores ativos economicamente. Mesmo com a presença de dor lombar crônica inespecífica, os trabalhadores continuam a exercer suas atividades profissionais. Este cenário não é raro em diferentes categorias profissionais. Indiscutivelmente a dor lombar está na lista dos problemas musculoesqueléticos com alta prevalência ${ }^{8,9}$.

A intensidade da dor dos trabalhadores do subsolo do carvão sintomáticos quanto ao segmento lombar se concentra entre leve e moderada, ou seja, representando respectivamente 50 e $30 \%$. Sobre estes achados diversas pesquisas procuram obter e estabelecer um ponto de corte correto para relacionar o grau de dor com aumento de risco na incapacidade em indivíduo com quadro álgico lombar. Esse ponto crucial de corte é considerado por alguns como grau de dor igual ou maior do que 5 como sendo este ponto, ou seja, destes valores para cima haveria relação proporcional com incapacidade ${ }^{10,11}$.

No entanto, mais recentemente este tipo de associação vem sendo contestada, sendo considerado como fator preditivo mais relevante a capacidade de manutenção e realização de atividade física e manutenção em movimento no trabalho, mesmo com a presença de dor ${ }^{12}$.

Dos 40 mineiros envolvidos no estudo, encontrou-se um percentual de $77,5 \%$ com fraqueza lombar quando comparados os valores em Kgf encontrados na avaliação dinamométrica com os valores referenciais de normalidade para o mesmo sexo masculino e faixas etárias. Este percentual mostrou-se surpreendentemente elevado, considerando um perfil trabalhador ativo e que demanda de significativo envolvimento físico para o exercício profissional no cotidiano. É patente na literatura a importância da força e do equilíbrio de forças musculares da região lombo pélvica para um bom funcionamento e manutenção tanto postural quanto da integridade físico-funcional deste segmento baixo da coluna ${ }^{13,14}$.

A condição funcional da coluna lombar é determinada pela condição estrutural (anatômica), pelo funcionamento sistêmico (fisiológico) e mecânico 


\section{Artigo Original}

\section{Saúde Funcional}

integrado (biomecânico) dos segmentos corporais. O equilíbrio lombo pélvico envolvendo os músculos multifídeo, iliocostal lombar, transverso do abdômen, quadrado lombar e diafragma, chamado de estabilização central, determinam a condição biomecânica deste segmento vertebral ${ }^{12}$.

Indivíduos com lombalgia mecânica tendem a apresentar fraqueza muscular envolvendo os músculos multífidos e transversos do abdômen associadas a uma estabilidade inadequada da região central que gera dor. Assim, a estabilização central ajuda a diminuir o quadro álgico e, consequentemente, interferir naquele ciclo vicioso que geralmente tem tendência a se instalar, ou seja, fraqueza que leva a instabilidade, que tende a gerar dor, que leva a fraqueza ${ }^{15}$.

De acordo com a CIF, a funcionalidade e a incapacidade podem ser descritas em três domínios de saúde, denominados Estrutura / Função do corpo, Atividade e Participação do indivíduo ${ }^{16}$.

A CIF tem como objetivo estabelecer uma padronização e unificação da linguagem para descrição de saúde e estados envolvendo a saúde. A funcionalidade está relacionada com uma abordagem biopsicossocial do indivíduo ${ }^{17}$. Conforme a CIF, nos domínios denominados estruturas e função do corpo, torna-se comum em pacientes com lombalgia encontrar-se alteração nesse domínio, como fraqueza e desequilíbrios musculares ${ }^{18}$. Dentro da CIF, a funcionalidade ainda engloba os níveis de participação social que demonstra a interação do sujeito na sociedade em situações de vida cultural ${ }^{19}$.

O nível de atividade física é uma variável que pode apresentar associação com incapacidade. Trabalhos que avaliaram indivíduos com dor lombar apontaram que a dor mostrou-se associada com menores níveis de atividade física ${ }^{20}$. Da mesma forma, outra pesquisa apresentou relação entre incapacidade e nível de atividade aeróbica em pacientes com dor lombar crônica, está se mostrando reduzida nestes indivíduos em relação a controles $^{21}$. A subjetividade do quadro doloroso musculoesquelético faz com que sejam difíceis os aprofundamentos quantitativos envolvendo a lombalgia ${ }^{22}$. Uma das principais características que envolvem a lombalgia crônica é a presença de incapacidade e consequente hipotrofia por desuso. A redução da capacidade funcional é um forte fator para o desenvolvimento de Lombalgia Crônica Inespecífica ${ }^{23}$. 
Pesquisas têm evidenciado que a diminuição na ativação da musculatura lombar tem levado a uma instabilidade do complexo lombo-pélvico e aumentado os acometimentos com quadros dolorosos.

Existe sustentação a partir de estudos controlados randomizados de que programas de exercícios melhoram especialmente os aspectos dor e função. Porém, o fato de grupos ativos contarem com menor probabilidade de sofrer de dor e incapacidade na região lombar em relação aos seus congêneres sedentários não determina que a realização de qualquer tipo de atividade física cotidiana, sem critérios, seja um fator de proteção para a coluna. Nesse sentido é determinante o tipo de exercício, o nível de atividade, a carga de trabalho, a postura corporal, entre outros fatores ${ }^{24}$.

Sabe-se que a frequência de atividade física do sujeito está associada com a incapacidade da pessoa. Em indivíduos que apresentavam quadro álgico lombar, ocorreu uma associação com frequência de atividade física menor ${ }^{20}$. A frequência de atividade física aeróbica, caracterizada como um exercício de longa duração, pode influenciar no aumento ou diminuição da incapacidade do cidadão com quadro álgico lombar $^{21}$.

No tratamento para a lombalgia crônica, autores relatam à importância de um programa envolvendo reforço muscular na reabilitação dessas pessoas. Assim, a fraqueza lombar fica mais evidente nesses pacientes com essa patologia ${ }^{25}$. Outras pesquisam mostram que a intervenção da Fisioterapia através de exercícios de fortalecimento na bola suíça voltados para pessoas com lombalgia crônica trouxe benefícios em suas vidas ${ }^{26}$.

A funcionalidade avaliada através do ODQ foi boa de forma generalizada em 97,5\% da amostra, que demonstrou resultados com incapacidade de inexistente a mínima, sendo que apenas 1 caso, representando 2,5\% da amostra, apresentou incapacidade moderada.

Estes achados em uma amostra trabalhadora ativa em termos laborais não mostraram correlação com dor, devendo-se considerar que o percentual de sintomáticos foi relativamente baixo, confirmando a lógica de que a presença de dor não determina diretamente a incapacidade. Este grupo trabalhador, incluindo os sintomáticos quanto à coluna lombar, encontra individualmente mecanismos de regulação neurofisiológica que faz com que a presença de sintomatologia dolorosa e 


\section{Artigo Original}

\section{Saúde Funcional}

a redução da força muscular lombar não reduza efetivamente sua capacidade para trabalhar.

No entanto, das duas principais variáveis analisadas neste estudo, quais sejam a funcionalidade e a força muscular lombar, a fraqueza da grande maioria dos mineiros destacou-se como uma tendência que merece atenção. De acordo com a $\mathrm{CIF}^{16}$ a funcionalidade está diretamente determinada pela condição da estrutura morfofisiológica, dos níveis de atividade, bem como sua participação social. Estas três dimensões preconizadas pela Classificação Internacional de Funcionalidade (CIF) poderão refletir nas melhores ou piores perspectivas decorrentes de controle sintomático e progressão funcional, objetivo este do paciente e dos profissionais de saúde que buscam o melhor manejo da lombalgia crônica inespecífica.

\section{CONCLUSÃO}

Sabe-se que a lombalgia crônica inespecífica tem como fator relevante sujeitos submetidos a esforços físicos elevados, como posturas estáticas frequentes, movimentos repetidos e levantamento de peso. Ela possui um predomínio na população com idade economicamente ativa, apresentando um início do quadro álgico lombar discreto, no qual tende a pior progressivamente com a mobilidade da região.

O presente estudo com trabalhadores do subsolo do carvão evidenciou que a fraqueza muscular está presente na grande maioria dos mineiros, independente da presença de dor lombar. A diminuição na força da musculatura lombar tende a gerar um ciclo vicioso que geralmente tende a se instalar, ou seja, fraqueza que leva à instabilidade, que tende a gerar dor, que leva a fraqueza.

Pode-se concluir que a redução da força dinamométrica lombar, embora não esteja associada à presença e intensidade de dor, bem como à incapacidade funcional, é um fator presente nesta amostra de mineiros do subsolo, podendo ser um elemento preditivo de acometimento do segmento lombar da coluna, com início de sintomatologia dolorosa e queda da capacidade funcional ou incapacidade moderada e até grave.

Os achados apontam para a necessidade de acompanhamento destas populações trabalhadoras visando à manutenção plena de suas condições 


\section{Artigo Original}

\section{Saúde Funcional}

funcionais e livres de afecções musculoesqueléticas da coluna lombar, que podem iniciar-se por sinais como redução da força.

\section{REFERÊNCIAS}

1. Lizier DT, Perez MV, Sakata RK. Exercícios para tratamento de lombalgia inespecífica. Rev bras anestesiol. 2012;62(6):842-6.

2. Helfenstein Junior M, Goldenfum MA, Siena C. Lombalgia ocupacional. Rev assoc med bras. 2010;56(5):583-9.

3. Braga AB, Rodrigues ACMA, Lima GVMP, Melo LR, Carvalho AR, Bertolini GRF. Comparação do equilíbrio postural estático entre sujeitos saudavéis e lombálgicos. Acta ortop bras. 2012;20(4):210-2.

4. Macedo DDP. Lombalgias. Cienc cult. 2011;63(2):42-4.

5. Meziat Filho N, Silva GA. Invalidez por dor nas costas entre segurados da Previdência Social do Brasil. Rev saúde pública. 2011;45:494-502.

6. Pires RAM, Sousa HA. Análise dos efeitos da tens, cinesioterapia e o método Mackenzie para redução da dor em pacientes com lombalgia. Universitas ciências saúde. 2012;10(2):127-35.

7. Medeiros BA, Vale EHM, Silva RGdS, Bezerra E. Efeitos do fortalecimento muscular sobre os níveis de dor e incapacidade funcional em indivíduos com lombalgia crônica. Rev novo enfoque. 2012;14(14):14-24.

8. Stefane T, Santos AM, Marinovic A, Hortense P. Dor lombar crônica: intensidade de dor, incapacidade e qualidade de vida. Acta paul enferm. 2013;26:1420.

9. Silva VS, Feno TB, Souza I, Rocha PECP. Dor lombar ocupacional: uma revisão de literatura. Rev dig efdeportes. 2013;181(18).

10. Jensen MP, Karoly P. Self-report scales and procedures for assessing pain in adults. In: Melzack DCTR, editor. Handbook of pain assessment. New York, NY, US: Guilford Press; 1992. p. 135-51.

11. Turner JA, Franklin G, Heagerty PJ, Wu R, Egan K, Fulton-Kehoe D, et al. The association between pain and disability. Pain. 2004;112(3):307-14.

12. Panjabi MM. Clinical spinal instability and low back pain. J electromyogr kinesiol. 2003;13(4):371-9.

13. Di Alencar TAM, Matias KFS, Bini RR, Carpes FP. Revisão etiológica da lombalgia em ciclistas. Rev bras ciênc esporte. 2011;33:507-28. 
14. Macedo CSG, Briganó JU. Terapia manual e cinesioterapia na dor, incapacidade e qualidade de vida de indivíduos com lombalgia. Espaç saúde (Online). 2009;10(2):1-6.

15. Prados MS, Di Naccio BL, Ferreira MC, Silva TCD. A efetividade dos exercícios de estabilização central na redução da dor lombar de origem mecânica. Uma revisão sistemática. Rev dig efdeportes (Online). 2012;175(17).

16. Cieza A, Stucki G. New approaches to understanding the impact of musculoskeletal conditions. Best pract res clin rheumatol. 2004;18(2):141-54.

17. Farias N, Buchalla CM. A classificação internacional de funcionalidade, incapacidade e saúde da organização mundial da saúde: conceitos, usos e perspectivas. Rev bras epidemiol. 2005;8:187-93.

18. Ocarino JM, Gonçalves GGP, Vaz DV, Cabral AAV, Porto JV, Silva MT. Correlação entre um questionário de desempenho funcional e capacidade física em pacientes com lombalgia. Braz j phys ther. 2009;13:343-9.

19. Sampaio RF, Mancini MC, Gonçalves GGP, Bittencourt NFN, Miranda AD, Fonseca ST. Aplicação da classificação internacional de funcionalidade, incapacidade e saúde (CIF) na prática clínica do fisioterapeuta. Rev bras fisioter. 2005;9(2):129-36.

20. Bjorck-van Dijken C, Fjellman-Wiklund A, Hildingsson C. Low back pain, lifestyle factors and physical activity: a population based-study. J rehabil med. 2008;40(10):864-9.

21. Smeets RJ, Wittink H, Hidding A, Knottnerus JA. Do patients with chronic low back pain have a lower level of aerobic fitness than healthy controls?: are pain, disability, fear of injury, working status, or level of leisure time activity associated with the difference in aerobic fitness level? Spine j. 2006;31(1):90-7.

22. Brazil AV, Ximenes AC, Radu AS, Fernades AR, Appel C, Maçaneiro CH, et al. Diagnóstico e tratamento das lombalgias e lombociatalgias. Rev bras reumatol. 2004;44:419-25.

23. Rasmussen CD, Jorgensen MB, Clausen $T$, Andersen LL, Stroyer J, Holtermann A. Does self-assessed physical capacity predict development of low back pain among health care workers? A 2-year follow-up study. Spine j. 2013;38(3):272-6.

24. Keen S, Dowell AC, Hurst K, Klaber Moffett JA, Tovey P, Williams R. Individuals with low back pain: how do they view physical activity? Fam pract. 1999;16(1):39-45.

25. Miateli Pires RA, Dumas FVL. Lombalgia: revisão de conceitos e métodos de tratamentos. Universitas ciências saúde. 2009;6(2):159-68. 
Artigo Original

Saúde Funcional

26. Araujo AGS, Oliveira L, Liberatori MF. Protocolo fisioterapêutico no tratamento da lombalgia. Cinergis. 2013;13(4):56-63. 